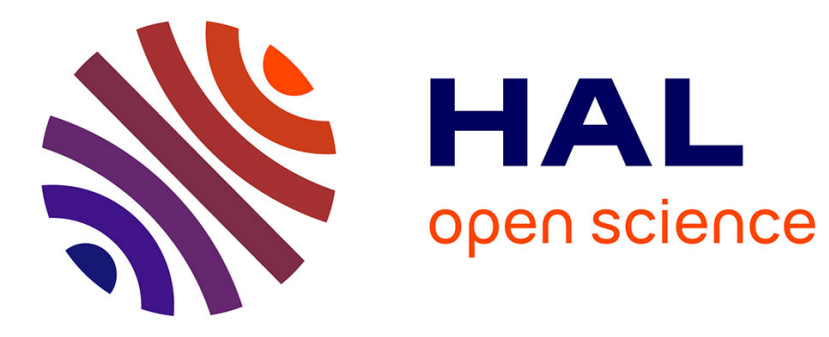

\title{
A new demodulation procedure for a class of multiplexed signals
}

Dilshad Surroop, Pascal Combes, Philippe Martin, Pierre Rouchon

\section{To cite this version:}

Dilshad Surroop, Pascal Combes, Philippe Martin, Pierre Rouchon. A new demodulation procedure for a class of multiplexed signals. IECON 2019 - 45th Annual Conference of the IEEE Industrial Electronics Society, Oct 2019, Lisbon, Portugal. 10.1109/IECON.2019.8927835 . hal-02413801

\section{HAL Id: hal-02413801 https://hal.science/hal-02413801}

Submitted on 16 Dec 2019

HAL is a multi-disciplinary open access archive for the deposit and dissemination of scientific research documents, whether they are published or not. The documents may come from teaching and research institutions in France or abroad, or from public or private research centers.
L'archive ouverte pluridisciplinaire HAL, est destinée au dépôt et à la diffusion de documents scientifiques de niveau recherche, publiés ou non, émanant des établissements d'enseignement et de recherche français ou étrangers, des laboratoires publics ou privés. 


\title{
A new demodulation procedure for a class of multiplexed signals
}

\author{
Dilshad Surroop ${ }^{1,2}$, Pascal Combes ${ }^{2}$, Philippe Martin ${ }^{1}$ and Pierre Rouchon ${ }^{1}$
}

\begin{abstract}
This paper introduces a set of estimators for a wide class of multiplexed signal. The signals of interest are decomposed on independent periodic signals with a shared frequency. This latter set of signals is as general as possible; that is, not necessarily orthogonal or sinusoidal. By an adequate linear combination of low-pass filters, we extract each of the components of the multiplexed signal with an arbitrary accuracy. Applications of this demodulation procedure include sensorless control of electrical machines using signal injection with the extraction of the ripple.
\end{abstract}

\section{NOMENCLATURE}

$C^{n} \quad$ Set of continuous functions with continuous first $n$ derivatives

$M^{1} \quad$ Moving average

$M^{k} \quad$ Moving average iterated k times

$P^{n} \quad$ nth-order moving average with phase shift compensation

$P_{i}^{n} \quad$ Operators for retrieving $y_{i}$ at order $\varepsilon^{n}$

PSD Power Spectral Density

$\left(s_{i}\right) \quad$ Set of 1-periodic independent signals

$\left(S_{i}\right)$ Gram-Schmidt's orthogonalization of $\left(s_{i}\right)$

$y$ Composite signal

$y_{i} \quad$ ith-coordinates of $y$ in the basis $\left(s_{i}\right)$

$\widetilde{y}_{i} \quad$ ith-coordinates in the orthogonal basis $\left(S_{i}\right)$

$\Delta_{\varphi}^{p} \quad$ pth-order backward difference of $\varphi$

$\varepsilon \quad$ Small parameter

$\check{\zeta}(t) \quad \zeta\left(\frac{t}{\varepsilon}\right)$

$\nu \quad$ Additive gaussian white noise

$A \lesssim B \quad$ There exists $K>0$ such that $A \leq K B$

$\langle f, g\rangle \quad \int_{0}^{1} f(\tau) g(\tau) d \tau$

$\overline{f^{2}} \quad\langle f, f\rangle$

$\|f\|_{\infty} \quad \sup \{|f(t)| ; t \in \mathbb{R}\}$

$\mathcal{O}_{\infty}(\varepsilon) \quad f(z, \varepsilon)=\mathcal{O}_{\infty}(\varepsilon)$ if there exists $K>0$ independent of $z$ and $\varepsilon$ s.t. $\|f(z, \varepsilon)\| \leq K \varepsilon$

\section{INTRODUCTION}

The design of causal filters for extracting information from measured signals with minimum time or phase lag is paramount in control applications. The need is particularly strong in sensorless control of electrical motors by signal injection. This technique, introduced in [1], [2] with sinusoidal signals, consists in adding a fast-varying periodic signal to the

\footnotetext{
1 D. Surroop, P. Martin and P. Rouchon are with the Centre Automatique et Systmes, MINES ParisTech, PSL Research University, Paris, France \{dilshad.surroop, philippe.martin, pierre.rouchon\}@mines-paristech.fr

${ }^{2}$ D. Surroop and P. Combes are with Schneider Toshiba Inverter Europe, Pacy-sur-Eure, France pascal.combes@se.com
}

control; this creates ripples in the measured currents which carry information about the rotor position. If this information is adequately decoded, it can be used to overcome low-speed observability issues, hence providing an effective means for controlling the motor, which is otherwise difficult. A rigorous analysis of the method with arbitrary injected signals was proposed in [3], with a simple general demodulation procedure; applications to electrical motors can be found in [4], [5]. Therefore, extracting with a good accuracy information from the periodic ripples in a signal is of major importance. The goal of this paper is to provide implementable estimators in a general framework encompassing signal injection. This type of demodulation is also of interest when considering the multiplexing of digital data. For instance, in Orthogonal FrequencyDivision Multiplexing (OFDM) technique introduced in [6], sequences of bits are encoded on orthogonal subcarriers; in this case the demodulation procedure consists of a fast Fourier transform [6], [7].

In this paper, we propose a general demodulation procedure for a composite signal that is encoded with known periodic signals. These periodic signals are functionally independent and are fast-varying with respect to the information to be decoded. We show that, with suitable linear combination of iterated moving averages, it is possible to extract all the components in the composite signal with an arbitrary accuracy. The estimators thus defined have a small time lag and are easy to implement on a programmable component.

The outline of the article is as follows: we first give an overview of the results in section II; we then provide detailed proofs in section III; finally, we illustrate in section IV the good behavior of the estimators on a numerical example.

\section{OVERVIEW OF THE MAIN RESULTS}

We consider a composite signal $y$ of the form

$$
y(t)=\sum_{i=0}^{N} y_{i}(t) s_{i}\left(\frac{t}{\varepsilon}\right)+\mathcal{O}_{\infty}\left(\varepsilon^{n}\right),
$$

where the $y_{i}$ 's are at least in $\mathcal{C}^{n}$, with bounded nth-order derivatives $y_{i}^{(n)}$; the $s_{i}$ 's are linearly independent 1-periodic functions, i.e. $\sum_{i=O}^{N} \lambda_{i} s_{i}(\tau)=0$ for all $\tau$ implies $\lambda_{i}=0$; $\varepsilon>0$ is a "small" positive parameter; $\mathcal{O}_{\infty}(\varepsilon)$ is the uniform "big O" of analysis. Intuitively speaking, the composite signal $y$ is a combination of the slowly-varying signals $y_{i}$ modulated by the fast-varying signals $s_{i}$. 
The goal is to retrieve the unknown $y_{i}$ 's from the measured $y$ and the known $s_{i}$ 's, with an accuracy of order $\varepsilon^{n}$, i.e. we want to design implementable estimators $P_{i}^{n}$ of $y_{i}$, such that

$$
P_{i}^{n}(y)(t)=y_{i}(t)+\mathcal{O}_{\infty}\left(\varepsilon^{n}\right), \quad 0 \leq i \leq N .
$$

These estimators are described in the general case in section III. In the simpler case of orthogonal $s_{i}$ 's -i.e. $\left\langle s_{i}, s_{j}\right\rangle=0$ for $i \neq j$, where $\langle f, g\rangle=\int_{0}^{1} f(\tau) g(\tau) d \tau$ denotes the usual scalar product-, they read for $n=1,2,3$

$$
\begin{aligned}
P_{i}^{1}(y)(t)= & \frac{1}{\overline{s_{i}^{2}}} M^{1}\left(y s_{i}\right)(t), \\
P_{i}^{2}(y)(t)= & \frac{1}{s_{i}^{2}}\left[2 M^{2}\left(y s_{i}\right)(t)-M^{2}\left(y s_{i}\right)(t-\varepsilon)\right], \\
P_{i}^{3}(y)(t)= & \frac{1}{\overline{s_{i}^{2}}}\left[\frac{17}{4} M^{3}\left(y s_{i}\right)-5 M^{3}\left(y s_{i}\right)(t-\varepsilon)\right. \\
& \left.+\frac{7}{4} M^{3}\left(y s_{i}\right)(t-2 \varepsilon)\right]
\end{aligned}
$$

where $\overline{s_{j}^{2}}=\left\langle s_{j}, s_{j}\right\rangle$ and the $M^{k}$,s are iterated moving averages (see nomenclature). The accuracy of the estimators improves with the order of the iterated moving averages, namely

$$
\begin{aligned}
& P_{i}^{1}(y)(t)=y_{i}(t)+\mathcal{O}_{\infty}(\varepsilon) \\
& P_{i}^{2}(y)(t)=y_{i}(t)+\mathcal{O}_{\infty}\left(\varepsilon^{2}\right) \\
& P_{i}^{3}(y)(t)=y_{i}(t)+\mathcal{O}_{\infty}\left(\varepsilon^{3}\right) .
\end{aligned}
$$

\section{THE DEMODULATION PROCEDURE}

This section details the design of the estimators $P_{i}^{n}$ in the general case, together with a proof of their accuracies.

\section{A. Orthonogalization of the $s_{i}$ 's}

The design of the filter relies on the decomposition of $y$ on an orthogonal basis (see lemma 2), which can be constructed using Gram-Schmidt orthogonalization process. For this, define $S_{0}=s_{0}$ and, for $1 \leq i \leq N$,

$$
S_{i}:=s_{i}-\sum_{j=0}^{i-1} \frac{\left\langle s_{i}, S_{j}\right\rangle}{\overline{S_{j}^{2}}} S_{j} .
$$

The set $\left(S_{i}\right)$ is orthogonal for this scalar product, and $\operatorname{span}\left(S_{i}\right)=\operatorname{span}\left(s_{i}\right)$. The coordinates $\widetilde{y}_{i}$ of $y$ on the new basis $\left(S_{i}\right)$ satisfy $\widetilde{y}_{N}=y_{N}$ and, for $1 \leq i \leq N$,

$$
\widetilde{y}_{N-i}(t)=y_{N-i}(t)+\sum_{j=N-i+1}^{N} \frac{\left\langle s_{j}, S_{N-i}\right\rangle}{\overline{S_{N-i}^{2}}} y_{j}(t) .
$$

The expression of $y$ in this orthogonal basis is then

$$
y(t)=\sum_{i=0}^{N} \widetilde{y}_{i}(t) S_{i}\left(\frac{t}{\varepsilon}\right)+\mathcal{O}_{\infty}\left(\varepsilon^{n}\right) .
$$

\section{B. Two preliminary results}

In this section, $M^{1}(\varphi)$ denotes the moving average of $\varphi$ with a window length of $\varepsilon$, and $M^{k}(\varphi)$ its k-times iteration. Namely, let $M^{0}(\varphi):=\varphi$ and

$$
M^{k}(\varphi)(t):=\frac{1}{\varepsilon} \int_{t-\varepsilon}^{t} M^{k-1}(\varphi)(\sigma) d \sigma, \quad k \geq 1 .
$$

We first recall a basic lemma on finite differences.

Definition 1: Let $\varphi$ be a continuous function. We define its pth-order $(p \in \mathbb{N})$ backward difference by

$$
\Delta^{p}(\varphi)(t):=\sum_{i=0}^{p}\left(\begin{array}{c}
p \\
i
\end{array}\right)(-1)^{i} \varphi(t-i \varepsilon)
$$

Lemma 1: Let $\varphi$ be $\mathcal{C}^{n}$ with $\varphi^{(n)}$ bounded. Then the pthorder backward difference of $\varphi^{(n-p)}$ satisfies the following inequality

$$
\left\|\Delta^{p}\left(\varphi^{(n-p)}\right)\right\|_{\infty} \lesssim \varepsilon^{p}\left\|\varphi^{(n)}\right\|_{\infty}, \quad p=0, \ldots, n .
$$

Proof: For $t \geq 0$ and $1 \leq i \leq p$, by Taylor-Lagrange's formula, there exists $t_{i} \in[t-i \varepsilon, t]$ such that

$$
\varphi^{(n-p)}(t-i \varepsilon)=\sum_{k=0}^{p-1}(-i \varepsilon)^{k} \frac{\varphi^{(n-p+k)}(t)}{k !}+(-i \varepsilon)^{p} \frac{\varphi^{(n)}\left(t_{i}\right)}{p !} .
$$

So the pth-order backward difference of $\varphi^{(n-p)}$ satisfies

$$
\begin{aligned}
\Delta^{p}\left(\varphi^{(n-p)}\right)(t)= & \sum_{k=0}^{p-1}(-\varepsilon)^{k} \frac{\varphi^{(n-p+k)}(t)}{k !} \sum_{i=0}^{p}\left(\begin{array}{c}
p \\
i
\end{array}\right)(-1)^{i} i^{k} \\
& +\frac{(-\varepsilon)^{p}}{p !} \sum_{i=0}^{p}\left(\begin{array}{c}
p \\
i
\end{array}\right)(-1)^{i} i^{p} \varphi^{(n)}\left(t_{i}\right) .
\end{aligned}
$$

Since for $0 \leq k \leq p-1, \sum_{i=0}^{p}\left(\begin{array}{c}p \\ i\end{array}\right)(-1)^{i} i^{k}=0$, we obtain

$$
\left\|\Delta^{p}\left(\varphi^{(n-p)}\right)\right\|_{\infty} \leq \varepsilon^{p}\left\|\varphi^{(n)}\right\|_{\infty} \sum_{i=0}^{p}\left(\begin{array}{c}
p \\
i
\end{array}\right) \frac{i^{p}}{p !} .
$$

We use this lemma to prove the following result, which is of major importance in the filter design.

Lemma 2: Let $\varphi$ be in $\mathcal{C}^{n}$ such that $\varphi^{(n)}$ is bounded, and $\zeta_{0}$ be a 1-periodic function with zero-mean. Then

$$
\left\|M^{n}\left(\varphi \check{\zeta}_{0}\right)\right\|_{\infty} \lesssim \varepsilon^{n}\left\|\varphi^{(n)}\right\|_{\infty}\left\|\zeta_{n}\right\|_{\infty},
$$

with $\zeta_{j+1}$ the zero-mean primitive of $\zeta_{j}$ and $\check{\zeta}(t):=\zeta\left(\frac{t}{\varepsilon}\right)$.

Proof: By induction, let's prove the following identity for $0 \leq m \leq n$

$$
M^{m}\left(\varphi \check{\zeta}_{0}\right)=\sum_{i=0}^{m}\left(\begin{array}{c}
m \\
i
\end{array}\right)(-\varepsilon)^{i} M^{i}\left(\Delta^{m-i}\left(\varphi^{(i)}\right) \check{\zeta}_{m}\right) .
$$

This expression is valid for $m=0$. Assume now it is true for $m \leq n-1$. Applying a single moving average to each of the terms in (5) and computing an integration by parts gives, for $0 \leq i \leq m$,

$$
\begin{aligned}
& M^{1} M^{i}\left(\Delta^{m-i}\left(\varphi^{(i)}\right) \check{\zeta}_{m}\right)=M^{i} M^{1}\left(\Delta^{m-i}\left(\varphi^{(i)}\right) \check{\zeta}_{m}\right) \\
& =M^{i}\left(\Delta^{m-i+1}\left(\varphi^{(i)}\right) \check{\zeta}_{m+1}\right)-\varepsilon M^{i+1}\left(\Delta^{m-i}\left(\varphi^{(i+1)}\right) \check{\zeta}_{m+1}\right) .
\end{aligned}
$$




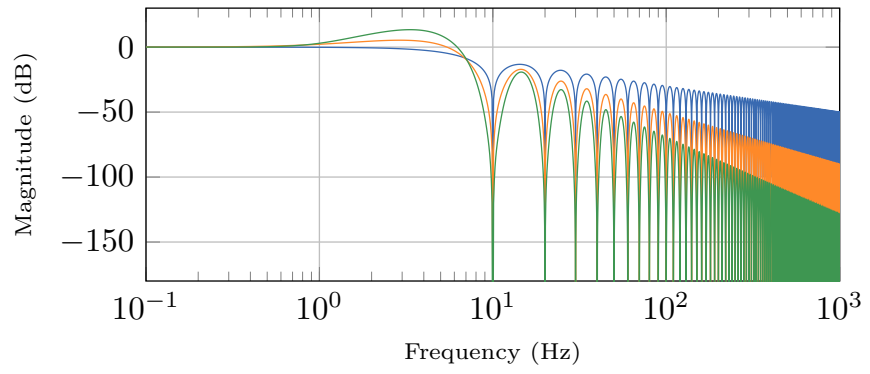

Fig. 1. Bode magnitiude plot of $P^{1}$ (blue), $P^{2}$ (orange) and $P^{3}$ (green)

Summing these terms, and applying Pascal's formula, we obtain the expected expression for $M^{m+1}\left(\varphi \check{\zeta}_{0}\right)$

$$
\begin{aligned}
& M^{m+1}\left(\varphi \check{\zeta}_{0}\right)=\sum_{i=0}^{m}\left(\begin{array}{c}
m \\
i
\end{array}\right)(-\varepsilon)^{i} \\
& \times\left[M^{i}\left(\Delta^{m-i+1}\left(\varphi^{(i)}\right) \check{\zeta}_{m+1}\right)-\varepsilon M^{i+1}\left(\Delta^{m-i}\left(\varphi^{(i+1)}\right) \check{\zeta}_{m+1}\right)\right] \\
&=\sum_{i=0}^{m+1}\left(\begin{array}{c}
m+1 \\
i
\end{array}\right)(-\varepsilon)^{i} M^{i}\left(\Delta^{m-i+1}\left(\varphi^{(i)}\right) \check{\zeta}_{m+1}\right),
\end{aligned}
$$

which concludes the induction. Besides, according to lemma 1,

$$
\left\|\Delta^{n-i}\left(\varphi^{(i)}\right) \check{\zeta}_{n}\right\|_{\infty} \lesssim \varepsilon^{n-i}\left\|\varphi^{(n)}\right\|_{\infty}\left\|\zeta_{n}\right\|_{\infty}, \quad 0 \leq i \leq n .
$$

This inequality holds when applying $M^{i}$ to the backward differences. That is

$$
\left\|M^{i}\left(\Delta^{n-i}\left(\varphi^{(i)}\right) \check{\zeta}_{n}\right)\right\|_{\infty} \lesssim \varepsilon^{n-i}\left\|\varphi^{(n)}\right\|_{\infty}\left\|\zeta_{n}\right\|_{\infty} .
$$

Using (5) with $m=n$, we eventually obtain

$$
\left\|M^{n}\left(\varphi \check{\zeta}_{0}\right)\right\|_{\infty} \lesssim \varepsilon^{n}\left\|\varphi^{(n)}\right\|_{\infty}\left\|\zeta_{n}\right\|_{\infty}
$$

\section{Design of the estimators}

The direct application of lemma 2 to $y \breve{S}_{i} i=1, \ldots, N$ gives

$$
\begin{aligned}
M^{n}\left(y \check{S}_{i}\right) & =\sum_{j=0}^{N} M^{n}\left(\widetilde{y}_{j} \check{S}_{j} \check{S}_{i}\right)+\mathcal{O}_{\infty}\left(\varepsilon^{n}\right) \\
& =M^{n}\left(y_{i}\left(\check{S}_{i}^{2}-\overline{S_{i}^{2}}\right)+y \overline{S_{i}^{2}}\right)+\mathcal{O}_{\infty}\left(\varepsilon^{n}\right) \\
& =\overline{S_{i}^{2}} M^{n}\left(\widetilde{y}_{i}\right)+\mathcal{O}_{\infty}\left(\varepsilon^{n}\right),
\end{aligned}
$$

since for $j \neq i, \check{S}_{j} \check{S}_{i}$ and $S_{i}^{2}-\overline{S_{i}^{2}}$ have zero mean. The orthogonality is used to isolate $\widetilde{y}_{i}$ from the other signals. Now we seek an estimate of $\widetilde{y}_{i}$ using only $M^{n}\left(y \check{S}_{i}\right)$. For this, we consider a linear combination of shifted $M^{n}$; a general result is the following theorem.

Theorem 1: Let $\varphi$ be in $\mathcal{C}^{n}$ such that $\varphi^{(n)}$ is bounded. There exists $\left(\alpha_{i}^{n}\right)_{0 \leq i \leq n-1}$ (specified in the proof) such that

$$
P^{n}(\varphi)(t):=\sum_{i=0}^{n-1} \alpha_{i}^{n} M^{n}(\varphi)(t-i \varepsilon)=\varphi(t)+\mathcal{O}_{\infty}\left(\varepsilon^{n}\right) .
$$

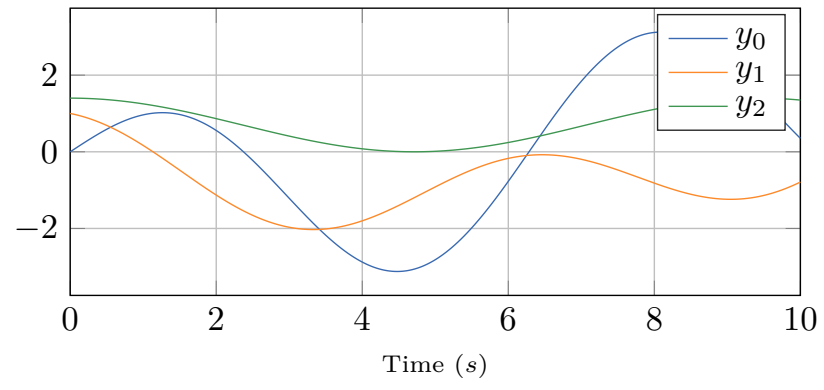

Fig. 2. The three components of $y$ : $y_{0}$ (blue), $y_{1}$ (orange) and $y_{2}$ (green)

Proof: Let first compute $M^{n}(\varphi)$. For a single moving average, and considering the Taylor expansion of $\varphi$, we have

$$
\begin{aligned}
M^{1}(\varphi)(t) & =\frac{1}{\varepsilon} \int_{0}^{\varepsilon} \varphi(t-\sigma) d \sigma \\
& =\frac{1}{\varepsilon} \int_{0}^{\varepsilon}\left[\sum_{i=0}^{n-1} \frac{(-\sigma)^{i}}{i !} \varphi^{(i)}(t)\right] d \sigma+\mathcal{O}_{\infty}\left(\varepsilon^{n}\right) \\
& =\sum_{i=0}^{n-1} \varepsilon^{i} a_{0, i}^{1} \varphi^{(i)}(t)+\mathcal{O}_{\infty}\left(\varepsilon^{n}\right),
\end{aligned}
$$

where $a_{0, i}^{1}$ satisfy, for $0 \leq i \leq n-1$,

$$
a_{0, i}^{1}:=\frac{(-1)^{i}}{(i+1) !}
$$

Let compute $M^{2}$ from the previous expression of $M^{1}$

$$
\begin{aligned}
& M^{2}(\varphi)(t)=\sum_{i_{1}=0}^{n-1} \varepsilon^{i_{1}} a_{0, i_{1}}^{1} M^{1}\left(\varphi^{\left(i_{1}\right)}\right)(t)+\mathcal{O}_{\infty}\left(\varepsilon^{n}\right) \\
& =\sum_{i_{1}=0}^{n-1} \varepsilon^{i_{1}} a_{0, i_{1}}^{1} \sum_{i_{2}=0}^{n-1-i_{1}} \frac{(-1)^{i_{2}}}{\left(i_{2}+1\right) !} \varphi^{\left(i_{1}+i_{2}\right)}(t)+\mathcal{O}_{\infty}\left(\varepsilon^{n}\right) \\
& =\sum_{i=0}^{n-1} \varepsilon^{i_{1}} a_{0, i}^{2} \varphi^{(i)}(t)+\mathcal{O}_{\infty}\left(\varepsilon^{n}\right),
\end{aligned}
$$

with $a_{0, i}^{2}$ defined for $0 \leq i \leq n-1$ by

$$
a_{0, i}^{2}:=\sum_{j=0}^{i} \frac{(-1)^{i-j}}{(i-j+1) !} a_{0, j}^{1}
$$

Iterating this process, we have the following expression

$$
M^{n}(\varphi)(t)=\sum_{i=0}^{n-1} \varepsilon^{i} a_{0, i}^{n} \varphi^{(i)}(t)+\mathcal{O}_{\infty}\left(\varepsilon^{n}\right),
$$

where $a_{0, i}^{n}$ is defined, for $0 \leq i \leq n-1$, by induction as

$$
a_{0, i}^{n}=\sum_{j=0}^{i} \frac{(-1)^{i-j}}{(i-j+1) !} a_{0, j}^{n-1}
$$




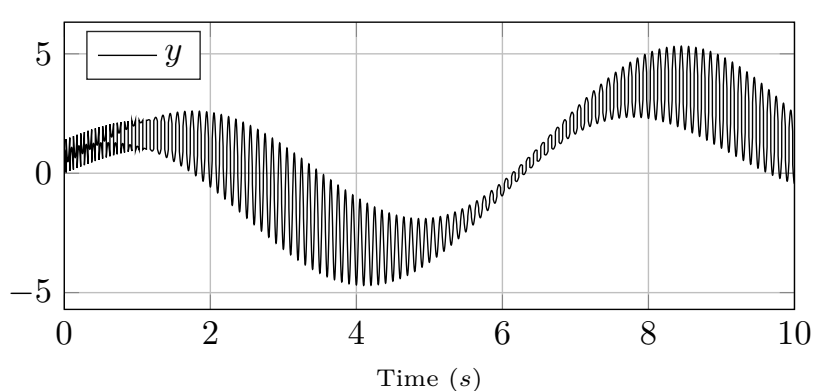

(a) Signal $y$

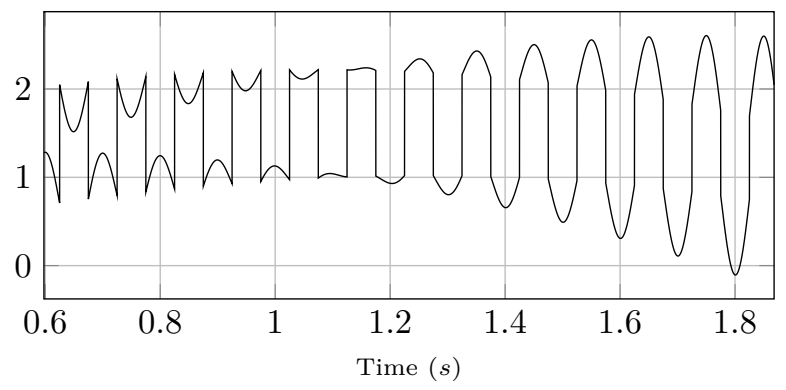

(b) Zoom on 3a

Fig. 3. The composite signal $y$

Now consider shifted $M^{n}$. Still by Taylor's expansion,

$$
\begin{aligned}
M^{n}(\varphi)(t-k \varepsilon) & =\sum_{i=0}^{n-1} \varepsilon^{i} a_{0, i}^{n} \varphi^{(i)}(t-k \varepsilon)+\mathcal{O}_{\infty}\left(\varepsilon^{n}\right) \\
& =\sum_{i=0}^{n-1} \varepsilon^{i} a_{k, i}^{n} \varphi^{(i)}(t)+\mathcal{O}_{\infty}\left(\varepsilon^{n}\right),
\end{aligned}
$$

with $a_{k, i}^{n}$ defined, for $0 \leq k, i \leq n-1$, by

$$
a_{k, i}^{n}=\sum_{j=0}^{i} \frac{(-k)^{i-j}}{(i-j) !} a_{0, j}^{n} .
$$

We define $\mathcal{M}^{n}(\varphi)(t)=\left(M^{n}(\varphi)(t-k \varepsilon)\right)_{0 \leq k \leq n-1}, \mathcal{A}^{n}=$ $\left(a_{k, i}^{n}\right)_{0 \leq k, i \leq n-1}$ and $\Phi(t)=\left(\varepsilon^{i} \varphi^{(i)}\right)_{0 \leq i \leq n-1}$. Then from the previous calculations we have

$$
\mathcal{M}^{n}(\varphi)(t)=\mathcal{A}^{n} \Phi(t)+\mathcal{O}_{\infty}\left(\varepsilon^{n}\right) .
$$

We assume $\mathcal{A}^{n}$ is invertible. Defining $\alpha^{n}=\left(\alpha_{i}^{n}\right)_{0 \leq i \leq n-1}$ such that $\alpha^{n} \mathcal{A}^{n}=\left(\begin{array}{llll}1 & 0 & \ldots & 0\end{array}\right)$, we get

$$
\alpha^{n} \mathcal{M}(t)=\varphi(t)+\mathcal{O}_{\infty}\left(\varepsilon^{n}\right) .
$$

Now defining the operator $P^{n}$ as follows, we finally have

$$
\begin{aligned}
P^{n}(\varphi) & :=\alpha^{n} \mathcal{M}(\varphi)(t)=\sum_{i=0}^{n-1} \alpha_{i}^{n} M^{n}(\varphi)(t-i \varepsilon) \\
& =\varphi(t)+\mathcal{O}_{\infty}\left(\varepsilon^{n}\right) .
\end{aligned}
$$

Combining these lemmas and theorem, we determine an expression of the estimate of each of the $y_{i}(0 \leq i \leq N)$

Corollary 1: Consider $y$ satifying (1), where $y_{i}(0 \leq i \leq$ $N)$ are $\mathcal{C}^{n}$ with $y_{i}^{(n)}$ bounded. Consider also the operator $P^{n}$

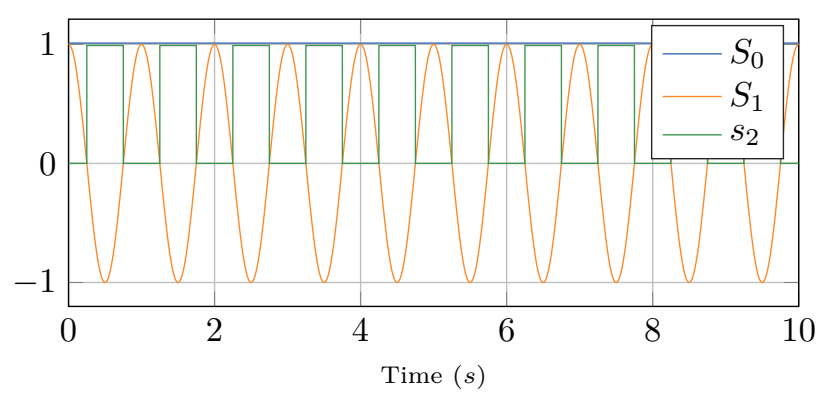

(a) $s_{0}=S_{0}, s_{1}=S_{1}, s_{2}$

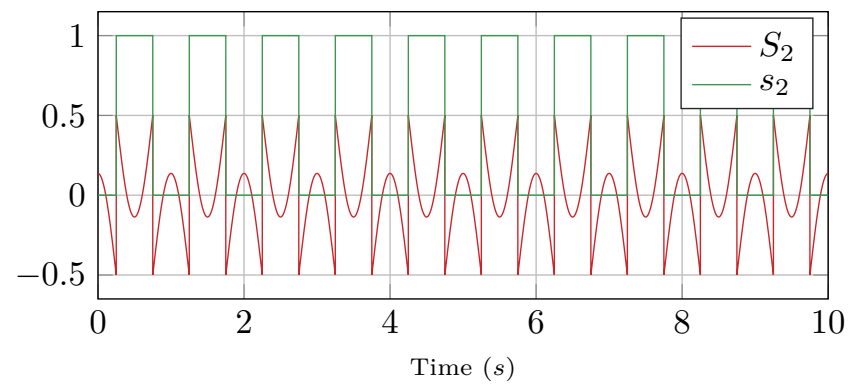

(b) $s_{2}$ and $S_{2}=s_{2}-\frac{1}{2}+\frac{2}{\pi} s_{1}$

Fig. 4. Signals $s_{0}, s_{1}, s_{2}$ and $S_{2}$ computed with Gram-Schmidt's process

defined in lemma 1. We define the operator $P_{i}^{n}$ such that it retrieves $y_{i}$ up to the nth-order in $\varepsilon$. Namely, for $1 \leq i \leq N$,

$$
\begin{aligned}
P_{N}^{n}(y) & :=\frac{1}{\overline{S_{N}^{2}}} P^{n}\left(y S_{N}\right)=y_{N}(t)+\mathcal{O}_{\infty}\left(\varepsilon^{n}\right), \\
P_{N-i}^{n}(y) & :=\frac{1}{\overline{S_{N-i}^{2}}} P^{n}\left(y S_{N-i}\right)-\sum_{j=N-i+1}^{N} \frac{\left\langle s_{j}, S_{N-i}\right\rangle}{\overline{S_{N-i}^{2}}} P_{j}^{n}(y) \\
& =y_{N-i}(t)+\mathcal{O}_{\infty}\left(\varepsilon^{n}\right) .
\end{aligned}
$$

Proof: According to lemmas 2 and 1 , since $S_{i} S_{j}$ has zero mean for $i \neq j$, we have

$$
\begin{aligned}
P^{n}\left(y \check{S}_{i}\right) & =\sum_{j=0}^{N} P^{n}\left(y_{j} \check{S}_{j} \check{S}_{i}\right)(t)+\mathcal{O}_{\infty}\left(\varepsilon^{n}\right) \\
& =\overline{S_{i}^{2}} \widetilde{y}_{i}+\mathcal{O}_{\infty}\left(\varepsilon^{n}\right) .
\end{aligned}
$$

With the relation given by Gram-Schmidt (4), we have the desired result.

\section{Sensitivity to noise}

In practical applications, the measurement $y$ is always corrupted by noise. Consider here the signal $y$ as in (1) with an additional gaussian white noise $\nu$ with a Power Spectral Density PSD $[\nu]$

$$
y(t)=\sum_{i=0}^{N} y_{i}(t) s_{i}\left(\frac{t}{\varepsilon}\right)+\nu+\mathcal{O}_{\infty}\left(\varepsilon^{n}\right) .
$$

This introduces an additive white noise $\nu_{i}^{n}$ in the expression of the estimate $P_{i}^{n}(y)$ of $y_{i}$. Specifically, the PSD of $\nu_{N}^{n}$ is

$$
\operatorname{PSD}\left[\nu_{N}\right](\omega)=\frac{1}{{\overline{S_{N}^{2}}}^{2}} \operatorname{PSD}\left[S_{N} \nu\right](\omega)\left|H^{n}(\jmath \omega)\right|^{2},
$$




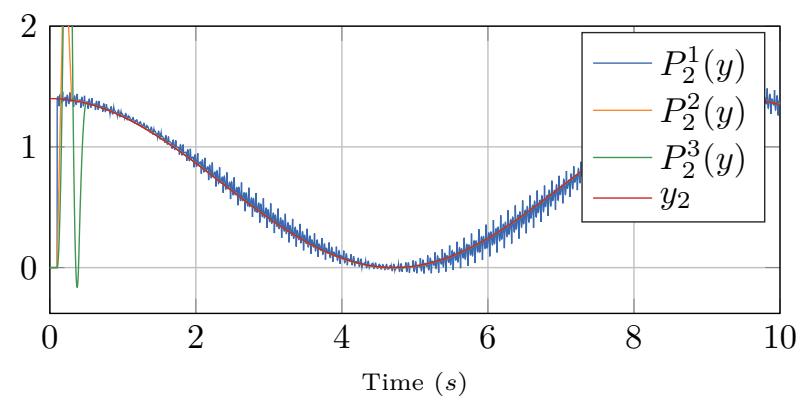

(a) $y_{2}, P_{2}^{i}(y)$

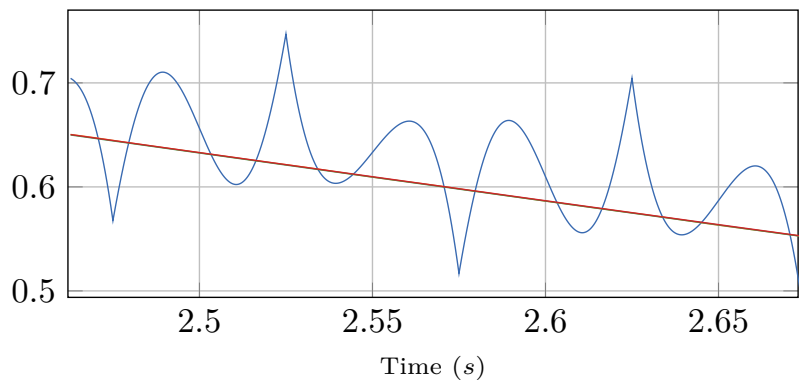

(b) Zoom on $5 \mathrm{a}$

Fig. 5. $y_{2}$ (red) and its estimation at order one $P_{2}^{1}(y)$ (blue), two $P_{2}^{2}(y)$ (orange) and three $P_{2}^{3}(y)$ (green) with $\varepsilon=0.1$

where $H^{n}$ is the transfer function of $P^{n}$ whose expression is $H^{n}(\jmath \omega):=\operatorname{sinc}^{n}\left(\frac{\epsilon \omega}{2}\right) \exp \left(\frac{-\jmath n \varepsilon \omega}{2}\right) \sum_{k=0}^{n-1} \alpha_{k}^{n} \exp (-\jmath k \varepsilon \omega)$.

Along the lines of [3], since $S_{N}$ and $\nu$ are independent, $\underline{S_{N}} \nu$ behaves as a gaussian white noise with $\operatorname{PSD}\left[S_{N} \nu\right]=$ $\overline{S_{N}^{2}} \mathrm{PSD}[\nu]$. The Bode plots of $H^{n}$, given in figure 1, show that the PSD of the noise is slightly amplified at low frequencies as $n$ increases.

The PSD of $\nu_{j}^{n}(j \leq N)$ can be computed in a similar manner. We define $s=\left(s_{i}\right)_{0 \leq i \leq N}$ and $S=\left(S_{i}\right)_{0 \leq i \leq N}$. GramSchmidt's process yields $s=\bar{B} S$ where $B=\left(b_{i j}\right)_{0 \leq i, j \leq N}$ is the transition matrix defined by

$$
b_{i j}= \begin{cases}\frac{\left\langle s_{i}, S_{j}\right\rangle}{\overline{S_{j}^{2}}} & \text { if } j \leq i \\ 0 & \text { otherwise. }\end{cases}
$$

Writing $B^{-1}=\left(\beta_{i j}\right)_{0 \leq i, j \leq N}$, we thus have for $0 \leq j \leq N$

$$
P_{j}^{n}(y)=\sum_{i=j}^{N} \frac{\beta_{i j}}{\overline{S_{i}^{2}}} P^{n}\left(y S_{i}\right) .
$$

Consequently the PSD of $\nu_{j}$ is

$$
\operatorname{PSD}\left[\nu_{j}\right](\omega)=\operatorname{PSD}\left[\left(\sum_{i=j}^{N} \frac{\beta_{i j}}{\overline{S_{i}^{2}}} S_{i}\right) \nu\right](\omega)\left|H^{n}(\jmath \omega)\right|^{2} .
$$

Following the previous calculations, we finally have

$$
\operatorname{PSD}\left[\nu_{j}\right](\omega)=\sum_{i=j}^{N} \frac{\beta_{i j}^{2}}{\overline{S_{i}^{2}}} \times \operatorname{PSD}[\nu](\omega)\left|H^{n}(\jmath \omega)\right|^{2} .
$$

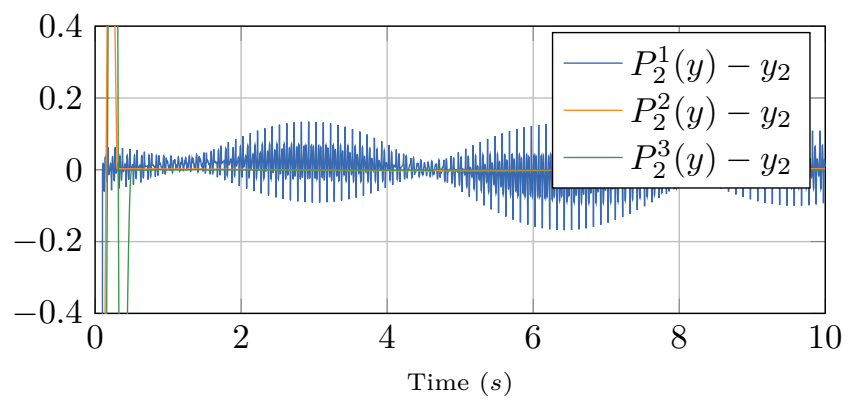

(a) $P_{2}^{i}(y)-y_{2}$

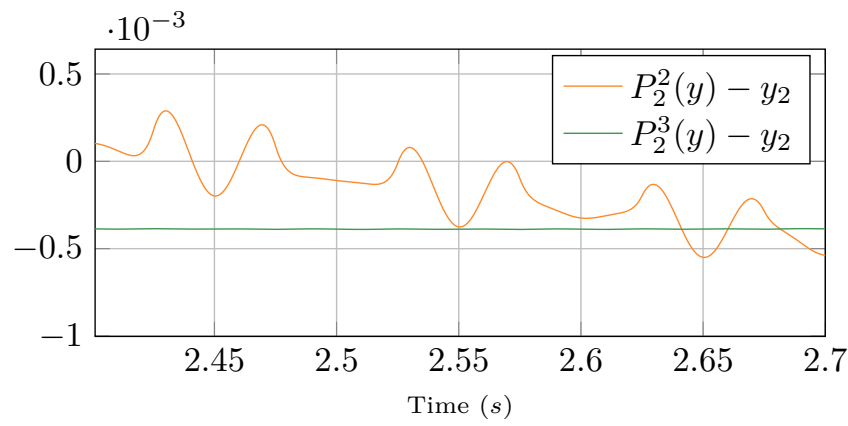

(b) Zoom on $6 \mathrm{a}$

Fig. 6. $\quad P_{2}^{n}(y)-y_{2}$ for $n=1$ (blue), 2 (orange), 3 (red) with $\varepsilon=0.1$

\section{A NUMERICAL EXAMPLE}

We now assess the previously described behaves well on a numerical example.

\section{A. Description of the scenario}

As an example, consider the composite signal

$$
y(t)=y_{0}(t) s_{0}\left(\frac{t}{\varepsilon}\right)+y_{1}(t) s_{1}\left(\frac{t}{\varepsilon}\right)+y_{2}(t) s_{2}\left(\frac{t}{\varepsilon}\right)+\mathcal{O}_{\infty}\left(\varepsilon^{3}\right),
$$

where $y_{0}, y_{1}, y_{2}$ are at least $C^{3}$ with $y_{i}^{(3)}$ bounded, and $s_{0}, s_{1}, s_{2}$ are 1-periodic and independent. Specifically, consider the three functions $y_{0}, y_{1}, y_{2}$

$$
\begin{aligned}
& y_{0}(t)=2 \sin (t)-1.5 \sin \left(\frac{t}{2}\right), \\
& y_{1}(t)=\cos (t)-1.2 \sin \left(\frac{t}{\pi}\right), \\
& y_{2}(t)=1.4 \cos ^{2}\left(\frac{t}{3}\right),
\end{aligned}
$$

shown in figure 2. The set of signals $s_{0}, s_{1}, s_{2}$ illustrated in figure $4 \mathrm{a}$ are defined on $t \in[0,1]$ by

$s_{0}(t)=1, \quad s_{1}(t)=\cos (2 \pi t), \quad s_{2}(t)= \begin{cases}1 & \text { if } \frac{1}{4} \leq t \leq \frac{3}{4} \\ 0 & \text { otherwise }\end{cases}$

The first step is to orthogonalize the set $\left(s_{0}, s_{1}, s_{2}\right)$ with GramSchmidt's process as described by (3). Define $S_{0}=s_{0}$; since $\left\langle 1, s_{1}\right\rangle=0$, define also $S_{1}=s_{1}$. For $S_{2}$, we have $\left\langle s_{2}, S_{0}\right\rangle=$ $\frac{1}{2},\left\langle s_{2}, S_{1}\right\rangle=-\frac{1}{\pi}$ and $\overline{S_{1}^{2}}=\frac{1}{2}$. Therefore, $S_{2}$ satisfies

$$
S_{2}(t)=s_{2}(t)-\frac{1}{2} s_{0}(t)+\frac{2}{\pi} s_{1}(t) .
$$




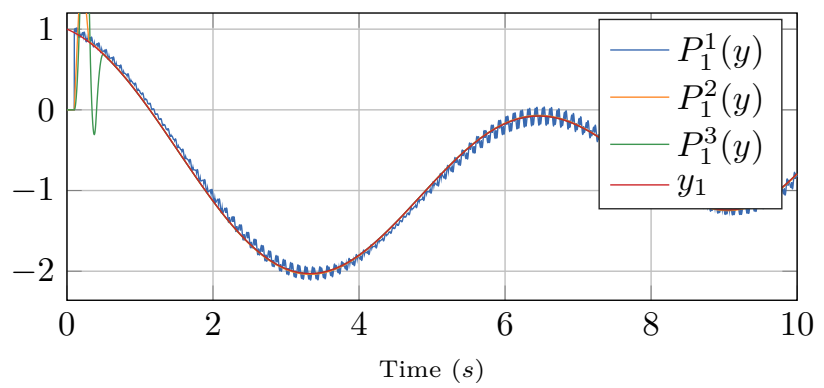

(a) $y_{1}, P_{1}^{i}(y)$

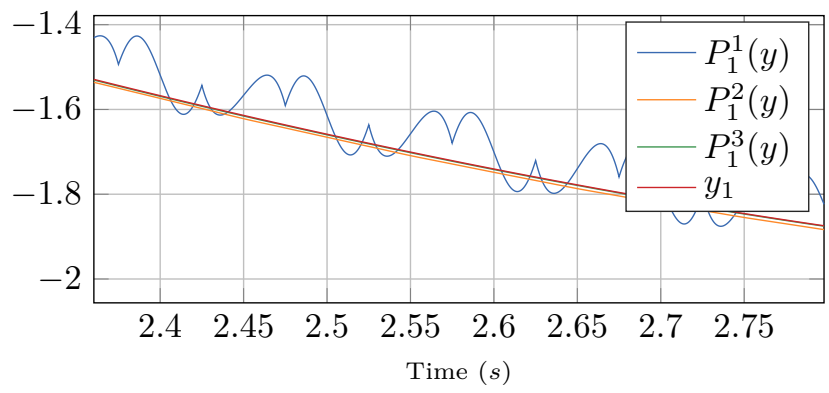

(b) Zoom on (a)

Fig. 7. $y_{1}$ and its estimation at order one $P_{1}^{1}(y)$, two $P_{1}^{2}(y)$ and three $P_{1}^{3}(y)$ with $\varepsilon=0.1$

The coordinates of $y$ in this new basis are, using (4)

$$
\begin{aligned}
& \widetilde{y}_{2}(t)=y_{2}(t), \\
& \widetilde{y}_{1}(t)=y_{1}(t)-\frac{2}{\pi} y_{2}(t), \\
& \widetilde{y}_{0}(t)=y_{0}(t)+\frac{1}{2} y_{2}(t) .
\end{aligned}
$$

The two signals $s_{2}$ and $S_{2}$ are represented in figure $4 \mathrm{~b}$. The composite signal $y$ can thus be rewritten as follows

$$
y(t)=\widetilde{y}_{0}(t) S_{0}\left(\frac{t}{\varepsilon}\right)+\widetilde{y}_{1}(t) S_{1}\left(\frac{t}{\varepsilon}\right)+\widetilde{y}_{2}(t) S_{2}\left(\frac{t}{\varepsilon}\right)+\mathcal{O}_{\infty}\left(\varepsilon^{3}\right) .
$$

Now we specify the expressions of the estimators $P_{i}^{n}$ for $n=1,2,3$ and $i=0,1,2$. For this, we compute the matrices $\mathcal{A}^{n}$ as defined in (6)

$$
\mathcal{A}^{1},=1 \quad \mathcal{A}^{2}=\left(\begin{array}{cc}
1 & -1 \\
1 & -2
\end{array}\right), \quad \mathcal{A}^{3}=\left(\begin{array}{ccc}
1 & -3 / 2 & 5 / 4 \\
1 & -5 / 2 & 13 / 4 \\
1 & -7 / 2 & 25 / 4
\end{array}\right) .
$$

Solving $\alpha^{n} \mathcal{A}^{n}=(1,0, \ldots 0)$ gives the values of the coefficients $\left(\alpha_{i}^{n}\right)$. It follows the expressions (2) for $P^{n}(n=1,2,3)$. Finally, corollary 1 provides the expression for each $y_{i}$

$$
\begin{aligned}
P_{2}^{n}(y) & :=\frac{1}{\overline{S_{2}^{2}}} P^{n}\left(y \check{S}_{2}\right), \\
P_{1}^{n}(y) & :=\frac{1}{\overline{S_{1}^{2}}} P^{n}\left(y \check{S}_{1}\right)+\frac{2}{\pi} P_{2}^{n}(y), \\
P_{0}^{n}(y) & :=\frac{1}{\overline{S_{0}^{2}}} P^{n}\left(y \check{S}_{0}\right)-\frac{1}{2} P_{2}^{n}(y) .
\end{aligned}
$$

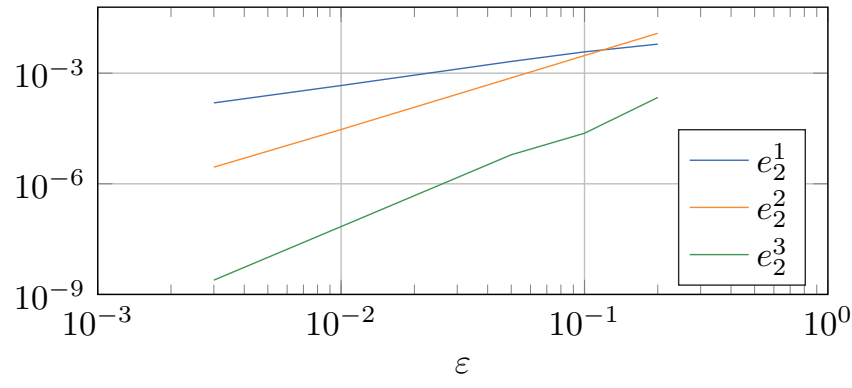

Fig. 8. RMS error $e_{2}^{n}$ for $n=1,2,3$ as a function of $\varepsilon$

\section{B. Discussion of the numerical results}

The simulations have been done in the time range $t \in$ $[0,10] s$ with $\varepsilon=0.1$, which is small enough compared to the rate of variation of the functions $y_{0}, y_{1}$ and $y_{2}$. We first retrieve $y_{2}$ using $P_{2}^{n}$. This estimate is then used to compute the estimation of $y_{1}$ and $y_{0}$ in accordance with the previous process. Figure 5 shows the function $y_{2}$ and its estimate $P_{2}^{n}(y)$ computed for $n=0,1,2$. The difference between $y_{2}$ and its estimates $P_{2}^{n}(y)$ is illustrated in figure 6. It appears that the orders of magnitiude of these differences are consistent with the inequality provided by lemma 2 : the amplitude $P_{2}^{n}(y)-y_{2}$ is approximately in $\varepsilon^{n}$. The initialization period of the filter is $n \varepsilon$, which explains the large error made by the estimators.

This estimate $P_{n}^{2}(y)$ is then used to retrieve $y_{1}$ (notice that it can be used to retrieve $y_{0}$ as well). The order of approximation of $y_{1}$ is still the same, as can be observed in figure 7 .

We repeat this simulation for different values of $\varepsilon$, and compute the RMS error $e_{2}^{n}=\sqrt{\int_{5}^{10}\left|y_{2}(\tau)-P_{2}^{n}(\tau)\right|^{2} d \tau}$ (we restrict the computation of the error on $t \in[5,10]$ to avoid the initialization part of the filters). Figure 8 shows the evolution of the $L^{2}$ error as a function of $\varepsilon$ in $\log$ scale. The slope of $e^{n}$ in log scale is equal to $n$ in accordance to corollary 1 .

\section{REFERENCES}

[1] P. Jansen and R. Lorenz, "Transducerless position and velocity estimation in induction and salient AC machines," IEEE Trans. Industry Applications, vol. 31, pp. 240-247, 1995.

[2] M. J. Corley and R. D. Lorenz, "Rotor position and velocity estimation for a salient-pole permanent magnet synchronous machine at standstill and high speeds," IEEE Transactions on Industry Applications, vol. 34, no. 4, pp. 784-789, 1998.

[3] P. Combes, A. K. Jebai, F. Malrait, P. Martin, and P. Rouchon, "Adding virtual measurements by signal injection," in American Control Conference, 2016, pp. 999-1005.

[4] A. K. Jebai, F. Malrait, P. Martin, and P. Rouchon, "Sensorless position estimation and control of permanent-magnet synchronous motors using a saturation model," International Journal of Control, vol. 89, no. 3, pp. 535-549, 2016.

[5] P. Combes, F. Malrait, P. Martin, and P. Rouchon, "Obtaining the current-flux relations of the saturated pmsm by signal injection," in Proceedings IECON 2017 - 43rd Annual Conference of the IEEE Industrial Electronics Society, vol. 2017-January, 2017, pp. 2097-2103.

[6] R. W. Chang, "Synthesis of band-limited orthogonal signals for multichannel data transmission," The Bell System Technical Journal, vol. 45, no. 10, pp. 1775-1796, Dec 1966.

[7] D. Tse and P. Viswanath, Fundamentals of Wireless Communication. Cambridge University Press, 2005. 